\title{
Electron scale structures of thin current sheets in magnetic reconnection
}

\author{
N. Jain ${ }^{1}$, A. S. Sharma ${ }^{1}$, L. M. Zelenyi ${ }^{2}$, and H. V. Malova ${ }^{2}$ \\ ${ }^{1}$ Dept. of Astronomy, University of Maryland, College Park, MD, USA \\ ${ }^{2}$ Institute for Space Research, Moscow, Russia \\ Correspondence to: N. Jain (njain@astro.umd.edu)
}

Received: 13 October 2011 - Revised: 19 December 2011 - Accepted: 5 January 2012 - Published: 3 April 2012

\begin{abstract}
An electron-magnetohydrodynamic model is used to simulate the structure of an electron scale current sheet during early phase of collisionless magnetic reconnection. The current sheet develops structures, viz. bifurcated, filamented and triple-peak structures at different locations in the current sheet. The reversal of the net out-of-plane electric field seen by electrons bifurcates the current sheet in the outflow regions, the individual peaks having scale sizes of a few electron skin depths. Secondary instabilities of the bifurcated CS lead to its filamentation in the outflow and separatrix regions while triple-peak structures form at reconnection sites. These structures have implications for the forthcoming NASA/MMS mission designed to resolve electron space and time scales in the magnetosphere.
\end{abstract}

Keywords. Magnetospheric physics (Magnetotail; Plasma waves and instabilities) - Space plasma physics (Magnetic reconnection)

\section{Introduction}

Thin current sheets $(\mathrm{CS})$ with thicknesses $\sim$ ion gyro-radius play important roles in the magnetosphere and have been identified by many satellite observations. These deviate significantly from the well-known Harris sheet and exhibit a variety of structures, viz. bifurcated (two peaks separated by a dip) (Runov et al., 2003; Hoshino et al., 1996) and triple peak structure (a central peak accompanied by two weaker side peaks) (Nakamura et al., 2006). Many theories and simulations have studied these non-Harris type structures. Hoshino et al. (1996) suggest that bifurcated CS corresponds to the slow shock structure associated with magnetic reconnection. While the ion current dominates in the centre of the magnetotail CS, a cross-field electron drift at the edges can support a double peaked structure (Asano et al., 2003). The ion current can bifurcate due to a gradual deterioration of the CS due to non-adiabatic scattering of quasi-trapped particles (Zelenyi et al., 2002).

Recently, current sheets with electron scale ( $\sim$ electron skin-depth, $\left.d_{\mathrm{e}}=c / \omega_{\mathrm{pe}}\right)$ non-Harris features, viz. bifurcated, triple peak and filamentary structure, have been observed by Cluster spacecraft (Wygant et al., 2005; Nakamura et al., 2006; Mozer et al., 2005). These observations have motivated theoretical and simulation studies on the development of the electron scale structures of the current sheets. The lower hybrid drift instability can produce an electrostatic potential structure across the sheet leading to an enhancement of the current (over its initial peak value) at the sides of the initial single peak, yielding a bifurcated structure (Daughton et al., 2004). However, this bifurcated structure is different from the one observed by Cluster (Wygant et al., 2005), in which the central dip of the bifurcated sheet reaches almost zero. Another mechanism of bifurcation is the disruption of the central peak caused by the trapping of current-carrying electrons in large amplitude electrostatic oscillations generated by current driven instabilities (Singh et al., 2006). The bifurcated sheets are, however, parallel to each other, in contrast to the cluster observations which show a diverging geometry. The triple peak structure formed in these simulations has two side peaks at the edges of the central peak, which is different from the observations (Nakamura et al., 2006) in which the side peaks are closer to the central peak and not at the edges.

Motivated by the Cluster observations on electron scales, which are usually associated with the magnetic reconnection events, and in view of the forthcoming NASA/MMS mission designed to probe electron space and time scales in the magnetosphere, simulation results on the development of various electron scale structures during early phase of reconnection are presented in this Letter. In collisionless plasmas, magnetic reconnection is initiated in electron scale current 


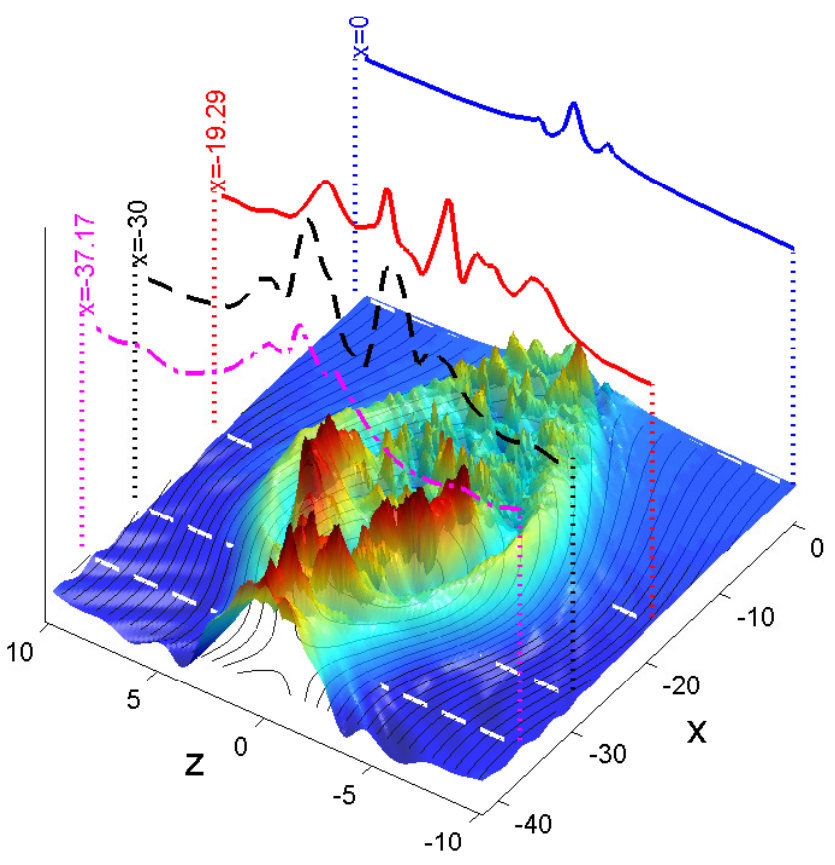

Fig. 1. The current density $J_{\mathrm{y}}$ (coloured surface) and magnetic field lines in the $\mathrm{x}-\mathrm{z}$ plane. The cuts of $J_{\mathrm{y}}$ along the white dashed lines on the $J_{\mathrm{y}}$-surface are also shown.

sheets and evolves from an early electron dynamics dominated time-dependent phase to a quasi-steady phase. An electron-magnetohydrodynamic (EMHD) model is used to describe the electron-scale processes in the early phase. It is shown that the bifurcation of an electron scale CS is an inherent feature of the reconnection, arising from the associated electron flows and electric field. In the nonlinear stage, the bifurcated CS gets filamented and triple peak structures form at the reconnection sites. All three types of structures are present simultaneously in reconnection.

A direct comparison of these structures with the Cluster observations may not be appropriate as the simulations pertain to the early stage of reconnection. However, it is possible that the structures, as observed by Cluster, formed in the early stage and then couple to ion scales in the later stage of the evolution retaining their shapes. The early stage electron dynamics and the cross scale coupling is planned to be probed by the forthcoming NASA/MMS mission, and the results presented here have important implications for such observations by providing the spatio-temporal development of the CS structure on electron scale and simultaneous presence of the different kinds of structures at different locations with respect to the reconnection site.

\section{EMHD model and structure of electron current sheets}

The EMHD model describes the motion of electron fluid in a charge neutralising background of stationary ions and is suitable for the early stage of reconnection. This model is valid for the space scales $<d_{\mathrm{i}}$ and time scales $<\omega_{\mathrm{ci}}^{-1}=$ $\left(e B_{0} / m_{\mathrm{i}} c\right)^{-1}$, and is represented by the equation (Kingsep et al., 1990)

$\frac{\partial}{\partial t}\left(\boldsymbol{B}-\nabla^{2} \boldsymbol{B}\right)=\nabla \times\left[\boldsymbol{v}_{\mathrm{e}} \times\left(\boldsymbol{B}-\nabla^{2} \boldsymbol{B}\right)\right]$,

where $\boldsymbol{v}_{\mathrm{e}}=-\nabla \times \boldsymbol{B}$. These equations assume uniform electron density and incompressibility (valid for $\omega_{\text {ce }}<\omega_{\text {pe }}$, which is usually satisfied in the magnetotail (Wygant et al., 2005)). The displacement current is ignored as $\omega \ll \omega_{\mathrm{pe}}^{2} / \omega_{\mathrm{ce}}$. In Eq. (1), the magnetic field is normalised by a typical value $B_{0}$, length by $d_{\mathrm{e}}$, time by $\omega_{\mathrm{ce}}^{-1}=\left(e B_{0} / m_{\mathrm{e}} c\right)^{-1}$ and velocity by the electron Alfvén velocity $v_{\mathrm{Ae}}=d_{\mathrm{e}} \omega_{\mathrm{ce}}$. In EMHD the frozen-in condition of magnetic field breaks down due to electron inertia, which is the dominant non-ideal term in generalised Ohm's law for scale sizes $\sim d_{\mathrm{e}}$ (Hesse and Winske, 1998).

The simulations are performed in the $x-z$ plane with the equilibrium profiles $\boldsymbol{B}_{0}=\tanh (z / L) \hat{x}$ and $\boldsymbol{J}_{0}=-\boldsymbol{v}_{\mathrm{e}}=$ $(1 / L) \operatorname{sech}^{2}(z / L) \hat{y}$. A CS scale $L=1.0$ is used. The boundary conditions are periodic along $x\left(-l_{\mathrm{x}}\right.$ to $\left.l_{\mathrm{x}}\right)$ while perturbations vanish at the $z$ boundaries $\left( \pm l_{\mathrm{z}}\right)$, with $l_{\mathrm{x}}=60$ and $l_{\mathrm{z}}=30$. The grid resolution is $\Delta x=\Delta z=0.15$ and time step is $\Delta t=0.01$. The simulations are initialised with small amplitude perturbations of the form $\tilde{f}(x, z)=$ $f_{1} \exp \left(-z^{2} / L^{2}\right) \Sigma_{m} \sin \left(m \pi x / l_{\mathrm{x}}\right)$ with $f_{1}=0.001$.

In the simulations, reconnection takes place at multiple sites with the dominant site at the centre $(x=z=0)$ and other secondary sites at different $x$-locations along the line $z=0$. Figure 1 shows a surface plot of $J_{\mathrm{y}}$ along with the magnetic field lines, reconnecting at $x=0$ (dominant site) and $x \approx-37$, in the fully nonlinear stage $(t=145)$. The cuts of $J_{\mathrm{y}}$ along $x=0,-19.29,-30$ and -37.17 are also shown. The region shown in Fig. 1 is symmetric around $x=0$ and focuses on a small region $(-37<x<0$ and $-10<z<10$ ) between the two reconnection sites at $x=0$ and $x \approx-37$ to highlight the main structures in $J_{\mathrm{y}}$. The two outer peaks which extend from $x=-37$ to $x=0$ parallel to the magnetic field separatrices form a pair of bifurcated sheets. In between the two outer peaks, two larger peaks originating from the site at $x \approx-37$ and extending towards $x>-37$ form another pair of bifurcated arms. Many small scale current filaments can be seen on the bifurcated sheets, which run parallel to the separatrices, and in the region between them.

The structures have different features depending on the location, as can be seen from the cuts of $J_{\mathrm{y}}$ in Fig. 1. At $x \approx 0$ and -37 , which are sites of reconnection, $J_{\mathrm{y}}$ has triple peak structure and the individual peaks have widths $\sim d_{\mathrm{e}}$. At $x=-30$, the current in the central region is bifurcated (thickness of individual peak $\sim 3 d_{\mathrm{e}}$ ) with two small peaks on the sides. The corresponding profile of $B_{\mathrm{x}}$ (not shown here) has a step-like structure at $z=0$. At $x=-19.29, J_{\mathrm{y}}$ has multiple peaks and consequently magnetic field profile has many step-like structures. 


\subsection{Bifurcated structure}

The complicated structure of $J_{\mathrm{y}}$, shown in Fig. 1, is a feature of the dynamic evolution of reconnection from an initial linear to fully nonlinear stage at $t=145$. The physics of the formation of the bifurcated structure can be studied in detail during the initial linear or weakly nonlinear stage of reconnection.

Figure 2a-d show the structures of $J_{\mathrm{y}}, v_{\mathrm{x}}, v_{\mathrm{z}}$ and $E_{\mathrm{y}}^{\prime}=$ $\left(\boldsymbol{E}+\boldsymbol{v}_{\mathrm{e}} \times \boldsymbol{B}\right)_{\mathrm{y}}$ in a smaller region around the central site $(x=z=0)$ in the initial weakly nonlinear stage (at $t=50$ ) of evolution, dominated by the maximally growing mode. In the outflow regions, the bifurcated sheets have diverging shapes with the outflow jets directed away from the reconnection site. The normal component $v_{\mathrm{z}}$ in Fig. 2c shows inflows to the reconnection region and diverging flows in the downstream regions. Since the magnetic field is frozen in the flow, the inflow leads to the thinning of the CS in the reconnection region. The diverging outflows, on the other hand, open up the CS, leading to the diverging shape of the CS in the outflow regions (Rogers et al., 2001). The reduction in the magnitude of $J_{\mathrm{y}}$ in the downstream regions leading to the bifurcation is due to the reversal of the net out-of-plane electric field seen by electrons in the downstream regions, as explained below.

The physical mechanism of the CS bifurcation is governed mainly by the out-of-plane $(y)$ component of the electron momentum equation:

$\frac{d v_{\mathrm{y}}}{d t}=-\left[E_{\mathrm{y}}+v_{\mathrm{z}} B_{\mathrm{x}}-v_{\mathrm{x}} B_{\mathrm{z}}\right]$

The changes in the current density are due to the terms on the right-hand side (RHS), which is the y-component of the total electric field $\boldsymbol{E}^{\prime}=\boldsymbol{E}+\boldsymbol{v}_{\mathrm{e}} \times \boldsymbol{B}$ seen by the electrons. The cuts along $z=0$ of $E_{\mathrm{y}}^{\prime}$ and various terms on RHS of Eq. (2) contributing to $E_{\mathrm{y}}^{\prime}\left(E_{\mathrm{y}}, v_{\mathrm{z}} B_{\mathrm{x}}\right.$ and $\left.v_{\mathrm{x}} B_{\mathrm{z}}\right)$ are shown in Fig. $2 \mathrm{e}$ at $t=50$. Near the line $z=0$, the second term $\left(v_{\mathrm{z}} B_{\mathrm{x}}\right)$ on the RHS is small for all values of $x$, since $v_{\mathrm{z}} \sim 0$ (by symmetry). The last term $\left(v_{\mathrm{x}} B_{\mathrm{Z}}\right)$ on the RHS is small near $x \approx 0$ (it is zero at the reconnection site as $B_{\mathrm{z}}, v_{\mathrm{x}}=0$ ). Therefore, in the vicinity of $x=0$, the reconnection electric field $E_{\mathrm{y}}$ in $+\hat{y}$ direction (going into the plane of the paper in Fig. 2a-d) accelerates electrons along $-\hat{y}$, leading to an intensification of the current along $+\hat{y}$. Away from $x=0, v_{\mathrm{x}}$ and $B_{\mathrm{Z}}$ increase and the Lorentz force term $v_{\mathrm{x}} B_{\mathrm{Z}}$ in Eq. (2) makes finite contribution to the total electric field. The total out-of-plane electric field $E_{\mathrm{y}}^{\prime}$ seen by electrons reverses its direction in the outflow regions where the nonlinear term $v_{\mathrm{x}} B_{\mathrm{z}}$ exceeds $E_{\mathrm{y}}$, as shown in Fig. 2e. It can be seen in Fig. 2d that $E_{\mathrm{y}}^{\prime}$ is along the equilibrium current in the central region while in the outflow region it is in the opposite direction, resulting in a bifurcation of the current there.

The structures in Fig. 2 form early in the simulation and their features can be understood in detail from the normal modes of the equilibrium, which is tearing unstable (Cali-
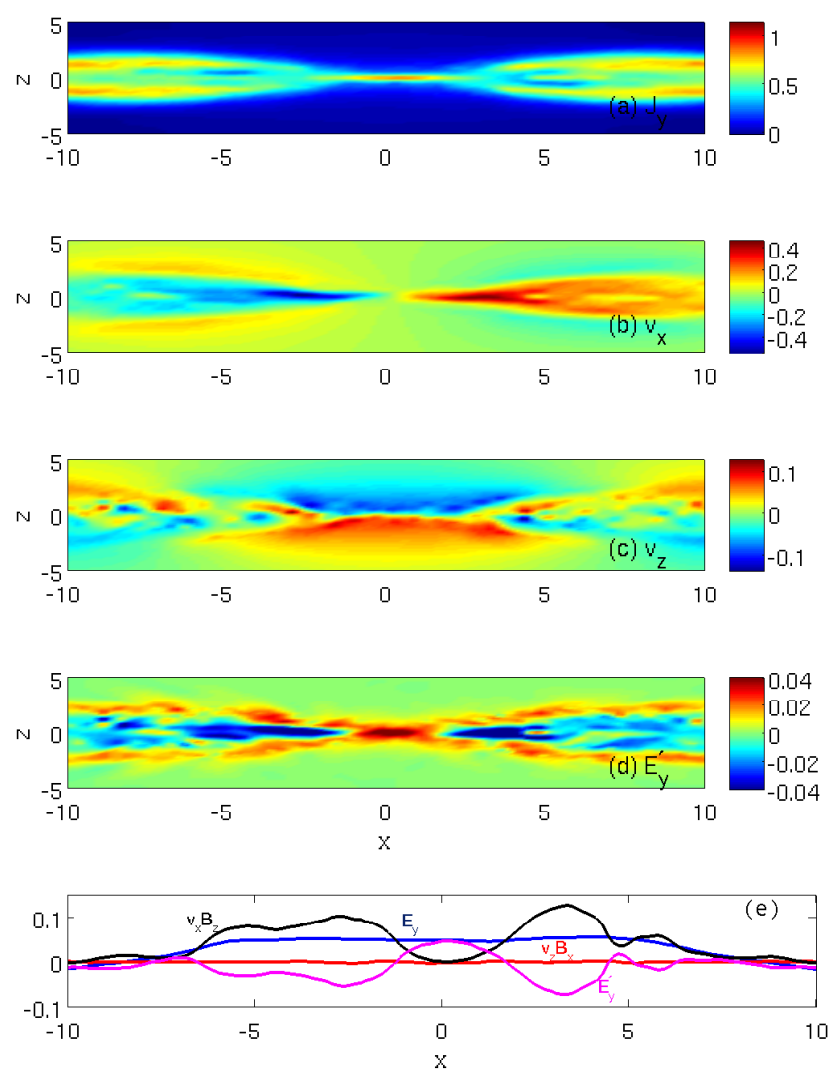

Fig. 2. The variables (a) $J_{\mathrm{y}}$, (b) $v_{\mathrm{x}}$, (c) $v_{\mathrm{z}}$ and (d) $E_{\mathrm{y}}^{\prime}=$ $\left[\mathbf{E}+\mathbf{v}_{\mathrm{e}} \times \mathbf{B}\right]_{\mathrm{y}}$ in the initial stage of simulations $(t=50)$. The cuts along $z=0$ of $E_{\mathrm{y}}^{\prime}$ (magenta), $v_{\mathrm{x}} B_{\mathrm{Z}}$ (black), $v_{\mathrm{Z}} B_{\mathrm{X}}$ (red) and $E_{\mathrm{y}}$ (blue) are shown in (e).

fano et al., 1999). The growth rates and eigen functions of the EMHD tearing mode are obtained from the numerical solution of the linearized EMHD eigen-mode equations yielding the maximally growing mode for $k_{\mathrm{x}} L=0.4 \Rightarrow \lambda_{\mathrm{x}} \approx 15$ for $L=1.0$. The real and imaginary parts of the corresponding eigen function $B_{\mathrm{z} 1}$ are shown in Fig. $3 \mathrm{a}$ and $\mathrm{b}$. To obtain two-dimensional structures in real space, we take $\tilde{B}_{\mathrm{z}}(x, z)=b_{\mathrm{z}}(z) \sin \left(k_{\mathrm{x}} x\right)$, where $b_{\mathrm{z}}(z)=\operatorname{real}\left(B_{\mathrm{z} 1}\right)$ (shown in Fig. 3a), allowing reconnection of the equilibrium field $\mathbf{B}_{0}=\tanh (z / L) \mathbf{x}$ at the site $x=z=0$. This gives $\tilde{B}_{\mathrm{x}}(x, z)=$ $\left(1 / k_{\mathrm{x}}\right) b_{\mathrm{x}}(z) \cos \left(k_{\mathrm{x}} x\right)$ and $\tilde{v}_{\mathrm{y}}(x, z)=\left(1 / k_{\mathrm{x}}\right) u_{\mathrm{y}}(z) \cos \left(k_{\mathrm{x}} x\right)$, where $b_{\mathrm{x}}(z)=b_{\mathrm{z}}^{\prime}(z)$, and $u_{\mathrm{y}}(z)=k_{\mathrm{x}}^{2} b_{\mathrm{z}}(z)-b_{\mathrm{z}}^{\prime \prime}(z)$ are shown in Fig. $3 \mathrm{c}$ and d. Figure $3 \mathrm{e}$ shows the structure of the CS when the perturbed current density $\tilde{J}_{\mathrm{y}}(x, z)=-\tilde{v}_{\mathrm{y}}(x, z)$ is added to the equilibrium current density $J_{\mathrm{y} 0}=\operatorname{sech}^{2}(z / L)$. Since $\tilde{J}_{\mathrm{y}}(x, z)>0$ in the vicinity of $z=0$ and $\tilde{J}_{\mathrm{y}}(x, z) \propto$ $\cos \left(k_{\mathrm{x}} x\right)$, the CS intensifies in the region of the length $\lambda_{\mathrm{x}} / 2$ $\left(-\lambda_{\mathrm{x}} / 4<x<\lambda_{\mathrm{x}} / 4, \lambda_{\mathrm{x}}=2 \pi / k_{\mathrm{x}}\right)$ and is bifurcated in the outflow regions, viz. $|x|>\lambda_{x} / 4$. For comparison, Fig. 3f shows the CS structure in the linear stage $(t=30)$ of the simulations when eigen structures begin to take shape from the initial perturbations. Figure $3 \mathrm{f}$ shows formation of the similar structure 

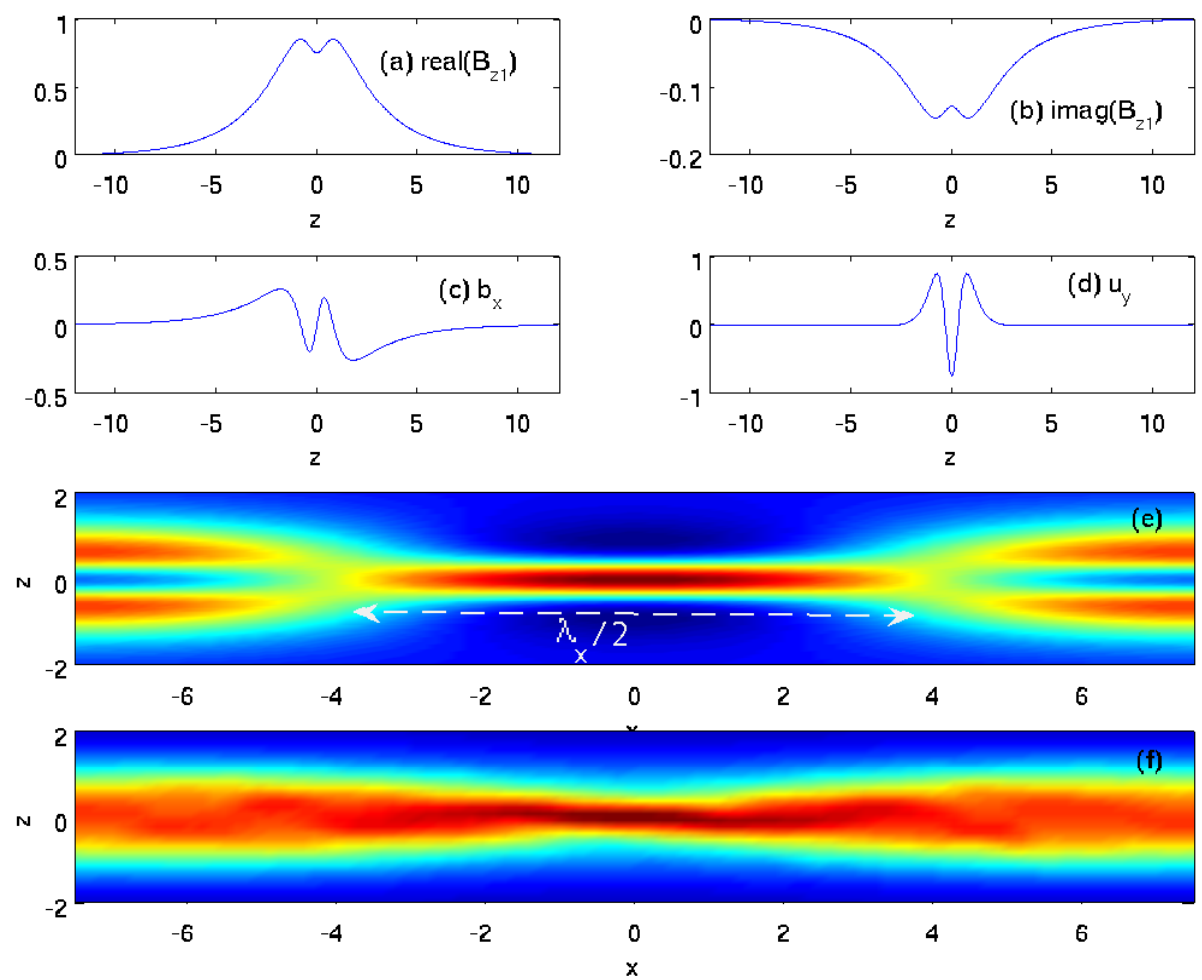

Fig. 3. The real (a) and imaginary (b) parts of the eigen function $B_{\mathrm{Z} 1}$ and the $z$-dependence of $\tilde{B}_{\mathrm{X}}(x, z)(\mathbf{c})$ and $\tilde{v}_{\mathrm{y}}(x, z)(\mathbf{d})$. The panel (e) shows the structure of the CS when perturbed current density $\tilde{J}_{\mathrm{y}}(x, z)=-\tilde{v}_{\mathrm{y}}(x, z)$ is added to the equilibrium current density $J_{\mathrm{y} 0}=\operatorname{sech}^{2}(x / L)$, while the bottom panel (f) shows the CS structure in the linear stage of the simulations.

as in Fig. 3e, however the bifurcation is not as clearly visible as in the latter. This is due to the perturbations in Fig. $3 \mathrm{f}$ being in the linear stage and, thus, have small amplitudes, while in Fig. 3e have amplitudes comparable to the equilibrium values.

It should be noted that the bifurcation in the linear theory is purely due to the reconnection electric field $E_{\mathrm{y}}$, as the nonlinear term $v_{\mathrm{X}} B_{\mathrm{Z}}$ is absent. This recognition leads to an understanding of the differences between the lengths of the reconnecting $\mathrm{CS}$ in the linear theory and simulations (Jain and Sharma, 2009). For $\tilde{B}_{\mathrm{z}} \propto \sin \left(k_{\mathrm{x}} x\right)$, Faraday's law gives $\tilde{E}_{\mathrm{y}} \propto \cos \left(k_{\mathrm{x}} x\right)$ which bifurcates the CS beyond $|x|>\lambda_{\mathrm{x}} / 4$ yielding CS length to be $\lambda_{\mathrm{x}} / 2$. However, in the simulations the nonlinear term $v_{\mathrm{x}} B_{\mathrm{z}}$ reverses the direction of $E_{\mathrm{y}}^{\prime}$ within a distance closer than $\lambda_{\mathrm{x}} / 2$, resulting in a length of reconnecting CS smaller than $\lambda_{\mathrm{x}} / 2 \sim 7.5$ (Fig. 2a).

In our simulations, the bifurcation of the CS in the downstream region is a consequence of reconnection facilitated by bulk electron inertia. This is an intrinsic feature of electron scale reconnection (in both linear and nonlinear stages), independent of the non-ideal effects breaking the frozen-in condition and has been observed in PIC simulations also (Daughton et al., 2006; Hesse et al., 1999). The basic origin of the bifurcation in the linear stage is the out-of-plane reconnection electric field (an intrinsic feature of reconnection) which, in the nonlinear stage, combines with the Lorentz force arising from the interaction of the electron outflow and the normal component of magnetic field, which are also intrinsic features of reconnection.

\subsection{Multi-peak and filamentary structures}

The CS shown in Fig. 1 exhibits more complicated structures, arising from the evolving reconnection. The reconnection at the dominant central site $(x=z=0)$ bifurcates the CS in the associated outflow regions. This leads to the formation of two separate peaks in the outflow regions, connected at $z \sim 0$, and appear as the outermost peaks in Fig. 1. The bifurcated current profile is susceptible to current shear instabilities (Jain et al., 2004) which not only break the bifurcated arms of the CS along the separatrices into filaments with scale size $\sim d_{\mathrm{e}}$, but also filament the CS in the outflow region between the separatrices. The filamentation of CS along separatrices is more clearly visible in the top panel of Fig. 4.

The triple peak structures, shown in Fig. 1, form at the reconnection sites due to the $E_{\mathrm{z}} \times B_{\mathrm{x}}$ drift of electrons in the inflow region. The in-plane Hall electric field $\boldsymbol{E}=\left(E_{\mathrm{x}}, E_{\mathrm{Z}}\right)$, shown by arrows around the central dominant site in the top panel of Fig. 4, points towards the CS in the inflow regions 
and, therefore, $E_{\mathrm{Z}} \times B_{\mathrm{x}}$ drift of electrons gives rise to two positive side peaks in $J_{\mathrm{y}}$. The bottom panel of Fig. 4 shows the cuts of $J_{\mathrm{y}}$ and $J_{\mathrm{y}, \text { drift }}=-E_{\mathrm{z}} / B_{\mathrm{x}}$ along $x=0$. The points near $z=0$ have been excluded in plotting $J_{\mathrm{y}, \text { drift }}$ as $B_{\mathrm{x}}=0$ at $z=0$. The profiles of $J_{\mathrm{y}}$ and $J_{\mathrm{y}, \text { drift }}$ match very well and the two side peaks in $J_{\mathrm{y}}$ coincide with those in $J_{\mathrm{y}}$,drift. A similar match is found for the triple peak structure at the secondary reconnection sites.

A new type of structure with four peaks (two side peaks along with the central region bifurcated into two peaks), seen at $x=-30$ in Fig. 1, forms due to the presence of the multiple reconnection sites with one of them being dominant. The two side peaks in the four-peak structure are the outer peaks in Fig. 1, which are the bifurcated peaks formed due to the reconnection at the central dominant site $(x=0)$. The field lines that reconnect at the dominant site $(x=0)$ reconnect again downstream forming the secondary sites, one of which, shown in Fig. 1 , is at $x \approx-37$ at $t=145$. The reconnection at this secondary site bifurcates the current sheet in the associated outflow regions and the bifurcated peaks appear as the two inner peaks of the four-peak structure.

\section{Discussion and conclusion}

The simulation results show that the electron processes in the early phase of collisionless reconnection leads to the complex structuring of the electron scale CS. Similar electron scale structures have been observed in the satellite observations, as part of the structures on larger space and time scales. The EMHD simulations presented here represent physics only on electron scale and caution is needed in a direct comparison with the observations. However, there are certain similarities in the shapes (not the sizes) of these electron scale structures between the observations and simulations, and here we point out these similarities. Motivated by these similarities, it is suggested that the electron scale CS structures begin to take shape in the early stage of reconnection and later couple to ion scales to become part of the larger scale structures. The data from the forthcoming NASA/MMS mission on the electron scale will enable direct comparison with the simulations.

A bifurcated CS with thickness of each sheet $\sim 3-5 d_{\mathrm{e}}$ was observed in the magnetotail (Wygant et al., 2005). In addition, the observed profile of $B_{\mathrm{x}}$ has many step-like structures near the centre of the CS, which corresponds to the multipeak structure of the CS. The cut of $J_{\mathrm{y}}$ at $x=-19.29$ in Fig. 1 shows a multi-peak profile of the current sheet with scale sizes of the individual peaks $\sim 1-3 d_{\mathrm{e}}$. These features of the CS compare well with the observations. However, it should be noted that the observed thin electron scale current sheet was embedded in a much thicker ion scale CS.

A triple peak CS with scale sizes of individual peaks down to $\sim 5 d_{\mathrm{e}}$ and a gross scale close to one ion skin depth $\left(d_{\mathrm{i}}\right)$ has been observed by Cluster in the magnetotail (Nakamura
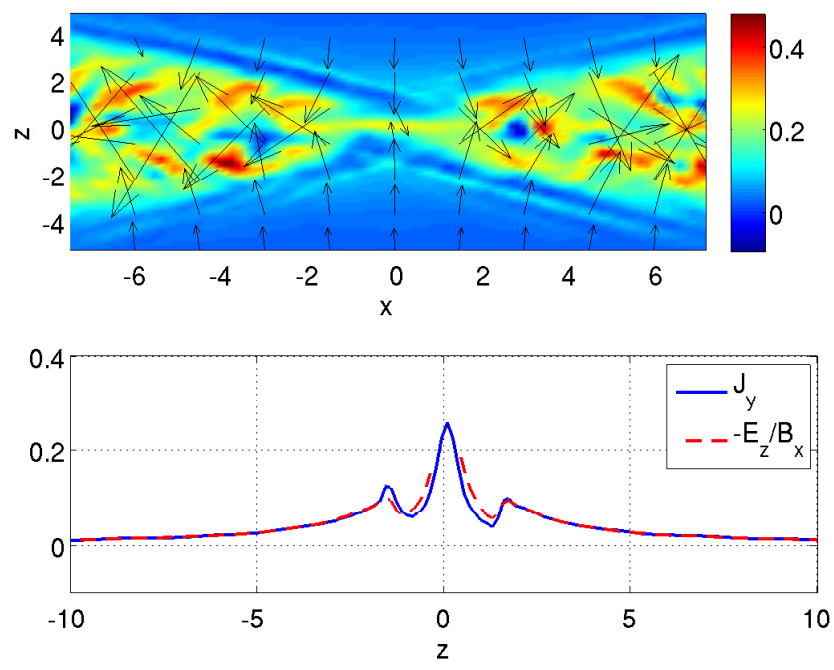

Fig. 4. Top panel: current density $J_{\mathrm{y}}$, shown by the colour, and inplane electric field vectors $\left(E_{\mathrm{X}}, E_{\mathrm{Z}}\right)$ by the arrows at $t=145$. Bottom panel: cuts of $J_{\mathrm{y}}$ and $-E_{\mathrm{Z}} / B_{\mathrm{X}}$ (points near $z=0$ excluded as $B_{\mathrm{X}}=0$ at $z=0$ ) along $x=0$.

et al., 2006). In our simulations, the triple-peak structure at the dominant site has side peaks at the edges of the central peak, while at the secondary site the side peaks develop closer to the central peak and its shape resembles the observed structure (Nakamura et al., 2006), indicating reconnection at multiple sites in the magnetotail. The number of the reconnection sites in the system is determined by the number of unstable wavelengths $(\lambda)$ that can fit in the length of the CS, i.e., by the ratio $l / \lambda$, where $l$ is the length of the CS. Since the wavelength of the most unstable mode is generally proportional to the CS thickness $L$, the condition for reconnection at multiple sites becomes $l / \lambda \propto l / L \gg 1$, which is readily satisfied in the magnetotail. The scale size $\sim 10 d_{\mathrm{e}}$ of the triple peak structure at the secondary site $(x \approx-37)$ is smaller than the one in the Cluster observations $\left(\sim d_{\mathrm{i}} \approx 40 d_{\mathrm{e}}\right)$. The error estimates in these Cluster measurements bring the scale size down to $60 \%$ smaller, viz. $16 d_{\mathrm{e}}$, which is closer to the case of the simulations.

In conclusion, the EMHD simulations of an electron scale CS show the development of a variety of structures during early phase of reconnection. The bifurcation of the CS in the outflow regions is an inherent feature of reconnection, as shown by the linear eigen-mode analysis and simulations. The mechanism for the bifurcation associated with reconnection is the reversal of the net out-of-plane electric field seen by electrons in the outflow region. The filamented CS can arise from secondary current shear instabilities (Jain et al., 2004) of the bifurcated CS, while triple peak structures form at the reconnection sites due to the $E_{\mathrm{z}} \times B_{\mathrm{x}}$ drift of electrons in the inflow regions. A new kind of structure at $x=-30$, Fig. 1, viz., bifurcated profile at the centre with two side peaks, is also seen in the simulations. Depending upon the 
trajectory of the spacecraft, one or more of the structures can be observed in a single reconnection event. The structures of the CS presented in this work are associated with reconnection and some of the features are consistent with the observations. These results have implications for the NASA/MMS and other missions designed to resolve electron scale structures in Earth's Magnetosphere.

Unlike most studies of reconnection, which are of its steady state, the results presented here are expected to be more prominent during the initial time dependent phase of reconnection after its onset in an electron scale CS and before ion dynamics become important. The structures formed during this initial phase may be modified by the ion dynamics, for example, the triple peak structure may be broadened due to the Hall electric field present over an extended inflow region in the presence of ion dynamics. As discussed in Sect. 2.1, the bifurcated structure can be understood in terms of the normal modes of the equilibrium and, thus, is expected to persist with some effects due to nonlinearity. The filamentary structures, on the other hand, are due to many processes whose time evolution can be influenced significantly by nonlinearity and ion dynamics.

Acknowledgements. This work was supported by NSFGEM postdoc Award No. 1027185 and NASA under Grant No. NNXO7AF42G.

Topical Editor R. Nakamura thanks one anonymous referee for her/his help in evaluating this paper.

\section{References}

Asano, Y., Mukai, T., Hoshino, M., Saito, Y., Hayakawa, H., and Nagai, T.: Evolution of the thin current sheet in a substorm observed by Geotail, J. Geophys. Res., 108, 1189, doi:10.1029/2002JA009785, 2003.

Califano, F., Prandi, R., Pegoraro, F., and Bulanov, S. V.: Twodimensional electron magnetohydrodynamic instabilities, Phys. Plasmas, 6, 2332-2339, 1999.

Daughton, W., Lapenta, G., and Ricci, P.: Nonlinear Evolution of the Lower-Hybrid Drift Instability in a Current Sheet, Phys. Rev. Lett., 93, 105004, doi:10.1103/PhysRevLett.93.105004, 2004.

Daughton, W., Scudder, J., and Karimabadi, H.: Fully kinetic simulations of undriven magnetic reconnection with open boundary conditions, Phys. Plasmas, 13, 072101, doi:10.1063/1.2218817, 2006.

Hesse, M. and Winske, D.: Electron dissipation in collisionless magnetic reconnection, J. Geophys. Res., 103, 26479-26486, 1998.
Hesse, M., Schindler, K., Birn, J., and Kuznetsova, M.: The diffusion region in collisionless magnetic reconnection, Phys. Plasmas, 6, 1781-1795, 1999.

Hoshino, M., Nishida, A., Mukai, T., Saito, Y., and Yamamoto, T.: Structure of plasma sheet in magnetotail: double-peaked electric current sheet, J. Geophys. Res., 101, 24775-24786, 1996.

Jain, N. and Sharma, A. S.: Electron Scale Structures in Collisionless Magnetic Reconnection, Phys. Plasmas, 16, 050704, doi:10.1063/1.3134045, 2009.

Jain, N., Das, A., and Kaw, P.: Kink instability in electron magnetohydrodynamics, Phys. Plasmas, 11, 4390, doi:10.1063/1.1777239, 2004.

Kingsep, A. S., Chukbar, K. V., and Yan'kov, V. V.: Reviews of Plasma Physics, vol. 16, Consultants Bureau, New York, p. 243, 1990.

Mozer, F. S., Bale, S. D., McFadden, J. P., and Torbert, R. B.: New features of electron diffusion regions observed at subsolar magnetic field reconnection sites, Geophys. Res. Lett., 32, L24102, doi:10.1029/2005GL024092, 2005.

Nakamura, R., Baumjohann, W., Asano, Y., Runov, A., Balogh, A., Owen, C. J., Fazakerley, A. N., Fujimoto, M., Klecker, B., and Reme, H.: Dynamics of thin current sheets associated with magnetotail reconnection, J. Geophys. Res., 111, A11206, doi:10.1029/2006JA011706, 2006.

Rogers, B. N., Denton, R. E., Drake, J. F., and Shay, M. A.: Role of dispersive waves in collisionless magnetic reconnection, Phys. Rev. Lett., 87, 195004, doi:10.1103/PhysRevLett.87.195004, 2001.

Runov, A., Nakamura, R., Baumjohann, W., Treumann, R. A., Zhang, T. L., Volwerk, M., Voros, Z., Balogh, A., Glabmeier, K.H., Klecker, B., Reme, H., and Kistler, L.: Current sheet structure near magnetic X-line observed by Cluster, Geophys. Res. Lett., 30, 1579, doi:10.1029/2002GL016730, 2003.

Singh, N., Deverapalli, C., and Khazanov, G.: Electrodynamics in a very thin current sheet leading to magnetic reconnection, Nonlin. Processes Geophys., 13, 509-523, doi:10.5194/npg-13-5092006, 2006.

Wygant, J. R., Cattell, C. A., Lysak, R., Song, Y., Dombeck, J., McFadden, J., Mozer, F. S., Carlson, C. W., Parks, G., Lucek, E. A., Balogh, A., Andre, M., Reme, H., Hesse, M., and Mouikis, C.: Cluster observations of an intense normal component of the electric field at a thin reconnecting current sheet in the tail and its role in the shock-like acceleration of the ion fluid into the separatrix region, J. Geophys. Res., 110, A09206, doi:10.1029/2004JA010708, 2005.

Zelenyi, L. M., Delcourt, D. C., Malova, H. V., and Sharma, A. S.: Aging of the magnetotail thin current sheets, Geophys. Res. Lett., 29, 1608, doi:10.1029/2001GL013789, 2002. 